\title{
Design and Evaluation of a Competitive Phosphorescent Immunosensor for Aflatoxin M1 Quantification in Milk Samples Using Mn:ZnS Quantum Dots as Antibody Tags
}

\author{
Sergio Forcada ${ }^{1}\left(\mathbb{D}\right.$, Adrián Sánchez-Visedo $^{2} \mathbb{D}$, Candela Melendreras ${ }^{2} \mathbb{D}$, Mario Menéndez-Miranda $^{1}$, \\ José M. Costa-Fernández ${ }^{2, *(\mathbb{D})}$, Luis J. Royo ${ }^{1}$ (D) and Ana Soldado ${ }^{2, *(D)}$ \\ 1 Department of Nutrition, Grasslands and Forages, Regional Institute for Research and Agro-Food \\ Development (SERIDA), 33300 Villaviciosa, Asturias, Spain; sforcada@serida.org (S.F.); \\ mmiranda@serida.org (M.M.-M.); ljroyo@serida.org (L.J.R.) \\ 2 Department of Physical and Analytical Chemistry, University of Oviedo, 33006 Oviedo, Asturias, Spain; \\ UO253767@uniovi.es (A.S.-V.); UO257805@uniovi.es (C.M.) \\ * Correspondence: jcostafe@uniovi.es (J.M.C.-F.); soldadoana@uniovi.es (A.S.)
}

Citation: Forcada, S.; Sánchez-Visedo, A.; Melendreras, C.; Menéndez-Miranda, M.; Costa-Fernández, J.M.; Royo, L.J.; Soldado, A. Design and Evaluation of a Competitive Phosphorescent Immunosensor for Aflatoxin M1 Quantification in Milk Samples Using Mn:ZnS Quantum Dots as Antibody Tags. Chemosensors 2022, 10, 41. https://doi.org/10.3390/ chemosensors10020041

Academic Editor: Guo-Hui Pan

Received: 8 December 2021

Accepted: 21 January 2022

Published: 24 January 2022

Publisher's Note: MDPI stays neutral with regard to jurisdictional claims in published maps and institutional affiliations.

Copyright: (C) 2022 by the authors. Licensee MDPI, Basel, Switzerland. This article is an open access article distributed under the terms and conditions of the Creative Commons Attribution (CC BY) license (https:// creativecommons.org/licenses/by/ $4.0 /)$.

\begin{abstract}
Aflatoxin M1 (AFM1) is one of the most widespread aflatoxins that can be present in the milk of lactating mammals. It can cause carcinogenicity, mutagenesis, teratogenesis, genotoxicity and immunosuppression. The WHO recommends reducing the AFM1 concentration in food products, so the European Commission has set a maximum allowable limit of $0.05 \mu \mathrm{g} \mathrm{L}^{-1}$ in milk and its products. Thus, there is a need to develop new methodologies to satisfy the demand for reliable, cost-effective, robust and sensitive AFM1 routine controls. In the present work, a competitive phosphorescent immunosensor for AFM1 quantification in milk, based on antibody-antigen recognition and Mn:ZnS quantum dots (d-QDs) as photoluminescent labels, has been developed. Two different assay strategies based on the use of d-QDs as labels of secondary antibodies (direct assay), or of a derivative species of the antigen AFM1-Bovine Serum Albumin (indirect assay) were compared in terms of analytical performance for AFM1 quantification. The best analytical results were obtained with the immunoassay format that uses d-QDs as tags of secondary antibodies (direct assay), and said design was finally selected. The selected immunosensor provided a detection limit for AFM1 quantification of only $0.002 \mu \mathrm{g} \mathrm{L}^{-1}$, which greatly satisfied the maximum tolerable limit of AFM1 in milk of $0.05 \mu \mathrm{g} \mathrm{L}^{-1}$. The accuracy, calculated as recovery of AFM1 in fortified skimmed milk samples, ranged from 81 to $90 \%$, with relative standard deviations from $3 \%$ to $14 \%$. These results bring to light the good performance of such phosphorescent biosensors as simple and fast alternatives to conventional chromatographic analytical methods.
\end{abstract}

Keywords: immunoassay; phosphorescence; quantum dot; aflatoxin M1

\section{Introduction}

Milk is essential for humans and perhaps the most valuable food for infants and elderly people, because of its nutritional and health benefits. That is why the safe consumption of milk is of the utmost importance for the European Union [1]. In this regard, one of the undesirable metabolites that can be found in milk are mycotoxins. These metabolites are produced by filamentous fungi that can contaminate animal feed, reaching milk produced for human consumption. Previous studies have confirmed that low levels of aflatoxins in the human diet is a risk for health; moreover, between $4.6 \%$ to $28.2 \%$ of reported hepatocellular carcinoma cases are due to aflatoxins exposure [2].

Aflatoxins are secondary metabolites produced by the Aspergillus flavus, Aspergillus parasiticus and Aspergillus nomius fungal species [3]. They are mainly found in cereals and crops which are included in dairy animals' feed. Aflatoxin B1 (AFB1) is the most prevalent and most toxic of aflatoxins. It is considered the most potent naturally occurring carcinogen 
with a Group 1 human carcinogen designation by the International Agency on Research on Cancer (IARC) [3,4]. In ruminants, after ingesting feed contaminated with AFB1, the AFB1 is transformed in a hydroxylated metabolite in the liver, the Aflatoxin M1 (AFM1), which is excreted in urine and secreted in milk. The AFM1 is a heat stable metabolite and cannot be eliminated or degraded by conventional milk processing procedures (i.e., pasteurization).

AFM1 causes carcinogenicity, mutagenesis, teratogenesis, genotoxicity and immunosuppression, specially targeting the liver and lungs $[4,5]$. Since milk is a daily consumed product in many countries, the risk of exposure to AFM1 is higher than that for AFB1, particularly in children. This public concern has motivated the enforcement of strict regulations by several countries in order to minimize the content of aflatoxins in food. On this matter, the FDA has set a maximum allowed level of $0.5 \mu \mathrm{g} \mathrm{L}{ }^{-1}$ of AFM1 in milk [6], but the European Commission has gone further by establishing a limit of $0.050 \mu \mathrm{g} \mathrm{L}^{-1}$ in raw and processed milk and $0.025 \mu \mathrm{g} \mathrm{L}{ }^{-1}$ of AFM1 in milk intended for lactating infants [1]. Consequently, high throughput routine controls for AFM1 in milk become essential.

High-performance liquid chromatography coupled to fluorescence detection (HPLCFD) [7-9] or coupled to mass spectrometry (HPLC-MS) [10-12] are currently used as standard methods for aflatoxin quantification. Although these methods allow the multiple quantification of aflatoxins in one experiment, they require extensive sample preparation to eliminate interferences, high-cost equipment and expert operators [4]. Additionally, the enzyme-linked immunosorbent assay (ELISA) is another method for AFM1 analysis which contributes to several commercial kits. Most ELISAs are specific, rapid and easy to use. However, these kits have limitations including cross-reactivity, lack of good recoverability and matrix interferences [4].

One of the greatest challenges facing the dairy industry is reducing the high-cost and time-consuming detection of AFM1. In this context, the combination of the high specificity of immunosensors with the growing field of nanotechnology offers great perspectives for detection of AFM1 at trace levels, overcoming some limitations of the abovementioned techniques. In this regard, photoluminiscent semiconductor nanocrystals, known as quantum dots (QDs), have replaced conventional fluorophores as tags in immunoassays [13-15] due to their high quantum yield, low photobleaching, high photochemical stability, size tunable emission, broad excitation spectra and easy surface modification [16,17]. The important advantages of fluorescence signal detection for bioassays, including a high sensitivity, low detection limits and short determination times are well recognized. However, fluorescence has some drawbacks when used in bioanalytical applications, mainly due to the scattering light and the autofluorescence from the biological media. Additionally, conventional QDs typically used as fluorophores in bioassays may exhibit an eventual toxicity derived from the nanoparticle core elemental composition (e.g., CdSe/CdTe). As an alternative, QDs exhibiting a long-lived photoluminescence (e.g., phosphorescence) and cores with low toxic elements become interesting as photoluminescence labels in immunoassays development. In this context, the use of phosphorescence-type emission as analytical signal allows the elimination of short-lived scattering light and background noise from the biological media, which would result in improved sensitivity and wider dynamic ranges of the nanoparticle-based immunoassay.

According to this, the intended addition of transition metal impurities in semiconductor quantum dots (doped quantum dots) constitutes an interesting approach for tuning quantum dots photoluminescence emission. As previously reported, doping ZnS quantum dots with Mn results in exceptional photoluminescence properties typical of phosphorescent emission, as a longer Stokes shift between excitation and emission and longer luminescent lifetimes in the order of $\mathrm{ms}[18,19]$. Longer dopant emission lifetime provides the opportunity to eliminate background fluorescence through time-resolved measurements. In this context, recent advances in controlling the synthesis and capping of such QDs have allowed well characterized, highly luminescent and aqueous-stable Mn-doped $\mathrm{ZnS}$ phosphorescent QDs to be obtained [18,20-22]. In fact, several analytical applications have been already successfully developed using Mn-doped QDs as luminescent labels [23-27]. 
Engineered nanostructures are highly valuable for multifunctional purposes such as signal transduction and amplification or molecular recognition. Unfortunately, one of the main limitations of nanoparticle-based techniques is the lack of reproducibility on the preparation of the bioconjugated materials. The morphology of nanomaterials may change from batch to batch and deviations are accumulated during multistep preparation procedures. The assurance of reproducibility requires an exhaustive control of the nanoparticle synthesis and functionalization process at several stages, ensured by using complementary analytical techniques that provide information. The nanoparticle concentration can be obtained by combining Inductively Coupled Plasma Mass Spectrometry (ICP-MS) with X-Ray Diffraction (XRD), the confirmation of bioconjugation effectiveness can be evaluated by using an Asymmetrical Flow Field-Flow Fractionation (AF4), the hydrodynamic radius by Dynamic Light Scattering (DLS) and the morphology of the QD and the antibody QD conjugate can be obtained by High-Resolution Transmission Electron Microscopy (HR-TEM).

In this work, two different immunoassay schemes using Mn-doped QDs (d-QDs) were designed and critically compared for sensitive detection and quantification of AFM1 in bovine milk. One scheme was based on the conjugation of phosphorescent dihydrolipoic acid-capped Mn:ZnS QDs to a derivative of the antigen (AFM1-BSA conjugate). In addition, a direct immunoassay scheme, in which d-QDs were bioconjugated with anti-IgG antibodies, was also designed and investigated. The approach showing the best analytical performance was then implemented in the development of an indirect competitive phosphorescence immunosensor for the quantification of AFM1 in milk samples.

\section{Materials and Methods}

\subsection{Reagents, Solutions and Materials}

All experiments were carried out with analytical-grade chemical reagents used as received without further purification. Deionized ultrapure water $(18.2 \mathrm{MU} / \mathrm{cm})$ was obtained with a Milli-Q system (Millipore, Bedford, MA, USA). Zinc sulfate heptahydrate, manganese chloride tetrahydrate, L-cysteine hydrochloride monohydrate, and standard solutions of $1000 \mathrm{mg} \mathrm{L}^{-1}$ of $\mathrm{Mn}, \mathrm{Zn}$ and S were obtained from MERCK (Darmstadt, Germany). Sodium sulfide nonahydrate, sodium hydroxide, lipoic acid, potassium tert-butoxide, $\mathrm{N}$-(3Dimethylaminopropyl)-N0-ethylcarbodiimide hydrochloride (EDC), $\mathrm{N}$-Hydroxysuccinimide (NHS), TWEEN 20, bovine serum albumin (BSA), aflatoxin M1 BSA conjugate, free aflatoxin M1 and methanol HPLC gradient grade were purchased from Sigma-Aldrich (Schnelldorf, Germany). Rat monoclonal anti-aflatoxin and goat polyclonal anti-rat antibody were obtained from Abcam (Cambridge, UK).

\subsection{Instrumentation}

QDs photoluminescent spectra were performed with the Varian Cary Eclipse Fluorescence Spectrometer (Agilent, Santa Clara, CA, USA) equipped with a xenon discharge lamp (peak power equivalent to $75 \mathrm{~kW}$ ), a Czerny-Turner monochromator and photomultiplier tube detector (Model R-298). The emission spectra (1 nm data interval), which presented a maximum emission wavelength at $590 \mathrm{~nm}$, were recorded upon excitation at $290 \mathrm{~nm}$, with a delay time of $0.2 \mathrm{~ms}$ and a gate time of $5 \mathrm{~ms}$. Excitation and emission slits were set at $10 / 10 \mathrm{~nm}$ respectively, and the averaging time selected to perform the experiments was $5 \mathrm{~ms}$. A microplate reader accessory was used for phosphorescent immunoassay measurements.

QDs characterization was carried out by simultaneous detection and quantification of the elements constituting the QD core ( $\mathrm{S}, \mathrm{Zn}$ and $\mathrm{Mn}$ ) using an ICP-MS/MS system (Agilent 8800 ICPQQQ, Tokyo, Japan). For separation and characterization of nanoparticles and their bioconjugates the ICP-MS/MS was coupled on-line to the AF4 system (AF2000, Postnova Analytics, Landsberg, Germany). Separation conditions are summarized in Table S1 (Electronic Supplementary Material, ESM). Dynamic light scattering spectra were measured by using a NanoZS90 instrument from Malvern Instruments, Houston, TX, USA. 


\subsection{Procedures}

\subsubsection{Synthesis of DHLA Capped Mn-Doped ZnS QDs}

Mn-doped ZnS QDs were synthesized as previously reported using a Mn:Zn precursors molar ratio of 3\% [18] to ensure optimum luminescent properties. To ensure a proper colloidal stability of the nanoparticles in aqueous and biological media, the surface of the synthesized QDs was modified with dihydrolipoic acid (DHLA), providing not only aqueous stabilization of the nanocrystals but also the capacity to be further bioconjugated to antibodies or biomolecules using conventional chemistry bioconjugation reactions.

The successful formation of the QDs was confirmed by Dynamic light scattering (DLS). A DLS spectrum of the product from the synthesis is provided in Figure S1 in the ESM. Results showed that product of the synthesis displayed a narrow nanoparticle-size distribution with a hydrodynamic diameter of $11 \mathrm{~nm}$.

\subsubsection{Bioconjugation Protocols and Purification}

One of the major drawbacks to achieve a routine use of inorganic nanoparticles as photoluminescence tags in immunosensing is, perhaps, the absence of "one bioconjugation reaction fits all", whereby one can link QDs to any biomolecule by following a reproducible scheme. Here, taking advantage of the surface modification of the QDs with DHLA ligands, a carbodiimide chemistry has been used for creating QDs bioconjugates based on the attachment the carboxyl groups of the QDs surface to amine groups of the antibodies. The bioconjugation reaction was carried out at room temperature with constant stirring for $2 \mathrm{~h}$ in $10 \mathrm{mM}$ pH 7.4 phosphate-buffered saline (PBS) containing $0.05 \%$ Tween 20 . Reaction conditions include the use of $66 \mathrm{nM}$ of the antibodies $\mathrm{Ab}, 2 \mu \mathrm{M}$ QDs (to ensure a QDs: $\mathrm{Ab}$ molar ratio of 30:1) and $\times 1500$ and $\times 3000$ molar excess EDC and NHS, respectively (the concentration of EDC and NHS were $3 \mathrm{mM}$ and $6 \mathrm{mM}$ respectively). During the first $10 \mathrm{~min}$ of stirring, QDs were mixed only with EDC and NHS in order to activate the carboxylic groups. After this initial time, the antibody was added until the completion of the reaction time. Finally, a purification step of the bioconjugate was performed by removing the unconjugated QDs and other excess of reagents by ultracentrifugation using a $100 \mathrm{kDa}$ cut-off centrifugal device (AMICON ${ }^{\circledR}$, Millipore, Madrid, Spain).

Similarly, a derivative of the AFM1 (AFM1-BSA from Sigma-Aldrich) was used here to facilitate the bioconjugation of the phosphorescent d-QDs to the analyte analog using the carbodiimide-mediated conjugation reaction. The reaction took place ensuring the same experimental conditions as those used for the conjugation of the antibody with the QDs; however, here different concentrations of the reactants were used: $110 \mathrm{nM}$ AFM1-BSA, $3.3 \mu \mathrm{M}$ QDs and $\times 1500$ and $\times 3000$ molar excess of EDC and NHS $(5 \mathrm{mM}$ and $10 \mathrm{mM}$, respectively). Considering the differences on the molecular weight of the AFM1-BSA and of the AFM1-BSA-QD, the unbound AFM1-BSA and other excess of reagents were removed by ultrafiltration (UHF) using a $100 \mathrm{KDa}$ cut-off membrane filter.

\subsubsection{Phosphorescent Immunoassay Formats Assayed}

A schematic representation of the different competitive immunoassays here developed and critically compared for AFM1 quantification is presented in Figure 1.

The phosphorescent QDs-based immunoassay formats assayed for AFM1 determination consisted of a competitive format, because AFM1 is a small molecule, having a single binding site with the antibody. Two different approaches have been investigated: an indirect competitive immunoassay, based on the phosphorescent QDs as tags of a derivative of the antigen (AFM1-BSA conjugate) and a direct competitive immunoassay, in which anti-IgG antibodies were labelled with the d-QDs.

In the indirect competitive assay, the competition was established via the binding between the target molecule (i.e., AFM1 from the sample) and the AFM1-BSA-QDs bioconjugate for the limited binding sites of the anti-AFM1 antibody previously immobilized in a microtiter plate. Here, the phosphorescent signal is inversely proportional to the concentration of AFM1 in the sample (Figure 1A). In this approach, the wells of the microtiter plates 
were coated with the anti-AFM1 antibodies $\left(100 \mu \mathrm{L}\right.$ of $2.5 \mu \mathrm{g} \mathrm{mL} \mathrm{m}^{-1}$ in $10 \mathrm{mM}$ PBS pH 7.4). The antibody solution was incubated at $37^{\circ} \mathrm{C}$ for $6 \mathrm{~h}$ and then overnight for $10 \mathrm{~h}$ at $4{ }^{\circ} \mathrm{C}$. This solution was removed and the plate was blocked with $150 \mu \mathrm{L}$ of BSA $(1 \% w / v)$. After blocking step, three washings of the wells were performed $(3 \times 150 \mu \mathrm{L}$ washing buffer $10 \mathrm{mM}$ PBS of $\mathrm{pH}=7.4$ containing $0.05 \%$ Tween 20 ).

A
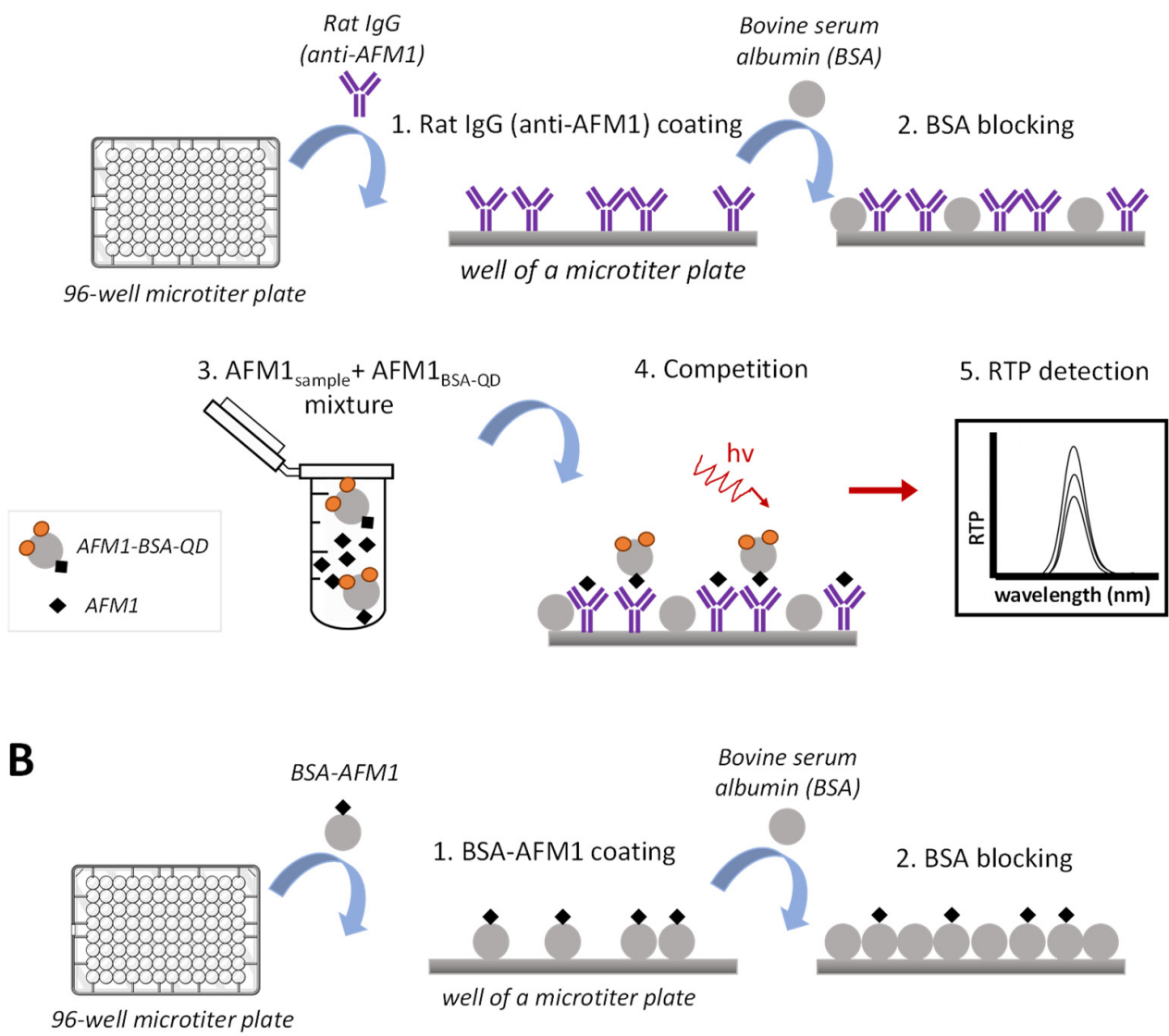

3. AFM1 + Rat IgG (anti-

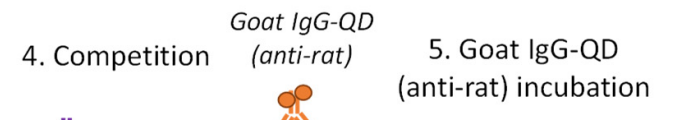

6. RTP detection
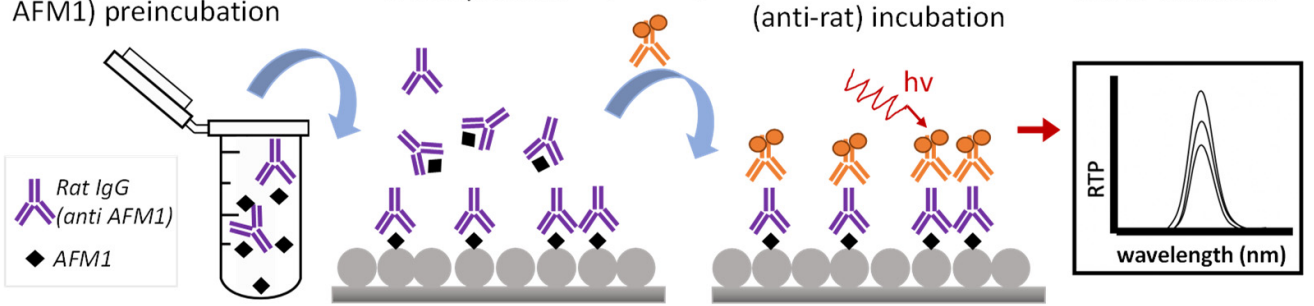

Figure 1. Schematic diagram of the competitive room temperature phosphorescent (RTP) immunoassays assayed for AFM1 quantification: (A) indirect competitive immunoassay; (B) direct competitive immunoassay.

The competitive assay was then based on an incubation of such microtiter plates with standards or samples containing AFM1 and labelled antigen. In a typical assay $50 \mu \mathrm{L}$ of the sample containing the AFM1 were mixed with the QD labelled BSA-AFM1 (50 $\mu \mathrm{L}$ of $1 \mu \mathrm{g} \mathrm{mL}^{-1}$ QD-BSA-AFM1) and the mixture was transferred to a well of the microtiter plate coated with the anti-AFM1 antibody and incubated for $2 \mathrm{~h}$ at $37^{\circ} \mathrm{C}$. Then, three washing steps were carried out before the room temperature phosphorescence (RTP) of each well was recorded. 
The direct competitive immunoassay was performed by tagging secondary anti-IgG antibodies with the QDs. Here, the wells of the microtiter plates were coated with the derivative of the antigen (BSA-AFM1) and the sample containing AFM1 and anti-AFM1 antibodies (in excess) are added to each of the wells. A competition to bind the antibodies is then established between the free AFM1 from the sample or the immobilized BSA-AFM1. After a washing step to remove the immunocomplex that is not immobilized, the anti-IgG QD-labelled antibody will bind the anti-AFM1 antibody bound to the immobilized BSAAFM1. The analytical signal will be inversely proportional to the initial AFM1 concentration in the sample (Figure 1B). Here, wells of a microtiter plate were coated with the BSA-AFM1 conjugate (i.e., $100 \mu \mathrm{L}$ of $1 \mu \mathrm{g} \mathrm{mL} \mathrm{m}^{-1}$ in $10 \mathrm{mM}$ PBS pH 7.4). The BSA-AFM1 solution was incubated at $37^{\circ} \mathrm{C}$ for $6 \mathrm{~h}$ and then overnight for $10 \mathrm{~h}$ at $4{ }^{\circ} \mathrm{C}$. The solution was removed and the plate was blocked with BSA $(150 \mu \mathrm{L} 1 \% w / v)$ for $1 \mathrm{~h}$ at $37^{\circ} \mathrm{C}$. After blocking, three washing steps of the wells were performed before the addition of follow-up solutions $(3 \times 150 \mu \mathrm{L}$ washing buffer $10 \mathrm{mM}$ PBS, $\mathrm{pH}=7.4$ containing $0.05 \%$ Tween 20$)$. Then, the sample to be analysed was incubated with anti-AFM1 rat antibody for $2 \mathrm{~h}$ at $37{ }^{\circ} \mathrm{C}(50 \mu \mathrm{L}$ of sample; $50 \mu \mathrm{L} 2 \mu \mathrm{g} \mathrm{mL}{ }^{-1}$ anti-AFM1 rat antibody). A total of $100 \mu \mathrm{L}$ of this solution was added into each well and incubated for $1 \mathrm{~h}$ at $37^{\circ} \mathrm{C}$. A further washing step was performed before adding $100 \mu \mathrm{L}$ of bioconjugate solution $\left(3 \mu \mathrm{g} \mathrm{mL}^{-1}\right.$, in terms of antibody conjugation) which was incubated for $1 \mathrm{~h}$ at $37^{\circ} \mathrm{C}$ ). Finally, after the corresponding washing steps, the room temperature phosphorescence (RTP) of each well was recorded as analytical signal.

Finally, the applicability of the developed immunoassay to real sample analysis was investigated by analyzing a commercial skimmed cow milk spiked with different levels of AFM1 in order to assess the analyte recoveries. Such commercial cow milk was selected due to the high similitude of its matrix to the real bovine milk and the absence of detectable amounts of AFM1.

It should be noted that it was not necessary to perform any sample pretreatment to the milk samples, except for a 10-fold milk dilution with PBS $10 \mathrm{mM}$, prior to their analysis. For calibration, AFM1 standards diluted in PBS $10 \mathrm{mM}$ were used.

\section{Results and Discussion}

\subsection{Synthesis and Characterization of the Antibody-QDs Bioconjugates}

The control of the bioconjugation process requires the knowledge of the QD concentration, that is, the number of nanoparticles present in a determinate volume. In this context, $\mathrm{Zn}$ content of the synthesized Mn-doped $\mathrm{ZnS}$ quantum dots, obtained by ICP-MS/MS analysis after digestion of aqueous aliquots from the QDs synthesis, was employed to calculate this concentration, as previously reported [20]. This information is essential to ensure reproducibility in successive bioconjugation reactions. Once the molar concentration of the nanoparticles had been calculated, the process of bioconjugation of the Mn-doped $\mathrm{ZnS}$ quantum dots to the anti-rat antibodies was optimized under the premise of maintaining the two desired properties, photoluminescence and molecular recognition. Several molar ratios $\mathrm{Ab}: \mathrm{QD}$ were assayed (from 1:5 to 1:40 molar ratios). It was observed that maximum phosphorescence signals were obtained when at least a 1:30 molar ratio was ensured (see Suplementary Figure S3).

The photoluminescence of the synthetized QDs-Ab conjugates was evaluated by measuring their RTP spectrum (see Figure 2). As can be seen in Figure 2, a detectable change on the excitation profile of the QDs has been observed after the attachment of antibodies to the QDs surface via covalent forces (bioconjugation process). Such a detectable change in the excitation spectra is of utmost interest as it could be used as evidence of the successful bioconjugation between the QDs and the antibodies. Even though such an unusual shift in the maximum excitation peak appears at $300 \mathrm{~nm}$, the bioconjugation process did not affect the phosphorescence emission from the free QDs used as antibody tags. In fact, the bioconjugate showed the same characteristic emission of free colloidal Mn-doped ZnS QDs, with a luminescent emission peak centered at $590 \mathrm{~nm}$ (Figure 2), being such issue of crucial value if the nanoplatform is intended to be used for bioanalytical purposes. 


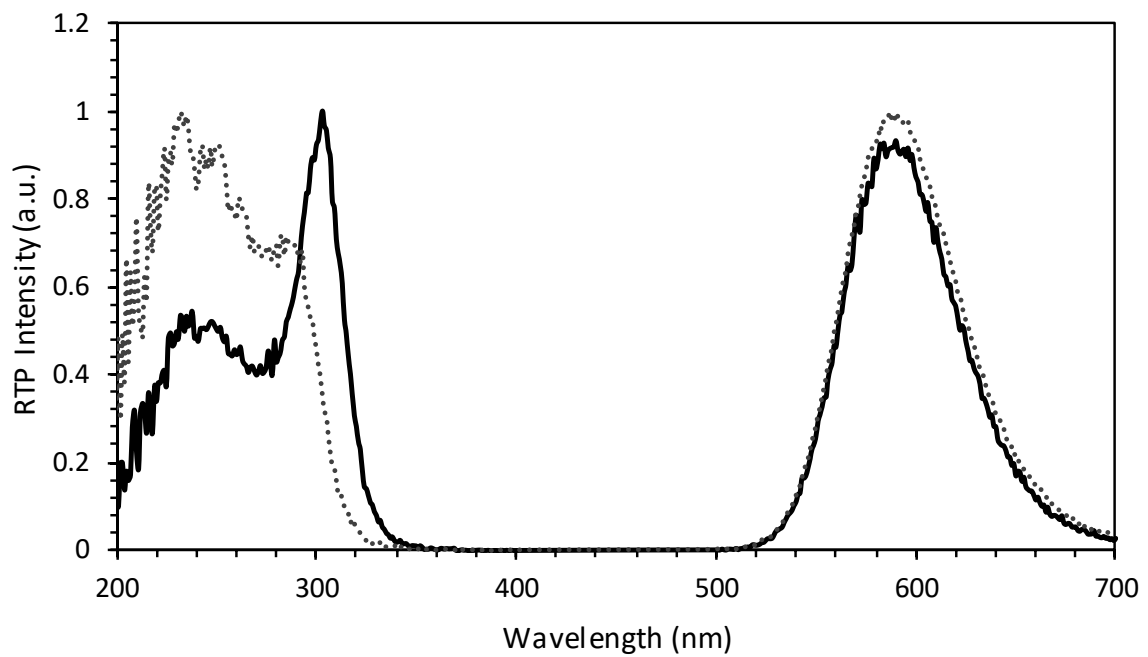

Figure 2. Photoluminiscent excitation/emission spectra of colloidal DHLA capped Mn:ZnS QDs (dotted line) and antibody-QD conjugate (solid line).

The assessment of the bioconjugation reaction between the quantum dots and the antibodies was carried out by the analysis of both the result of the bioconjugation mix and the free QDs solution using the asymmetric flow field-flow fractionation (AF4) nanoparticleseparation system coupled on-line to an ICP-MS/MS detector. The AF4 conditions are summarized in the Electronic Supplementary Information (ESI) section, while the obtained fractograms (of the analysis of free colloidal QDs and the bioconjugation mixture) are shown in Figure 3. The elution profile of the free QDs shows a sole peak having the maximum at $10.66 \mathrm{~min}$, which corresponds to a single population of nanoparticles containing $\mathrm{Zn}$ and Mn. The bioconjugation mixture was injected under the same separation conditions. Then, a single peak appears with a maximum at $11.94 \mathrm{~min}$ and, thus, shifted to a longer retention time in comparison with the free QDs. This confirms the actual formation of a link between the QDs and the antibodies.

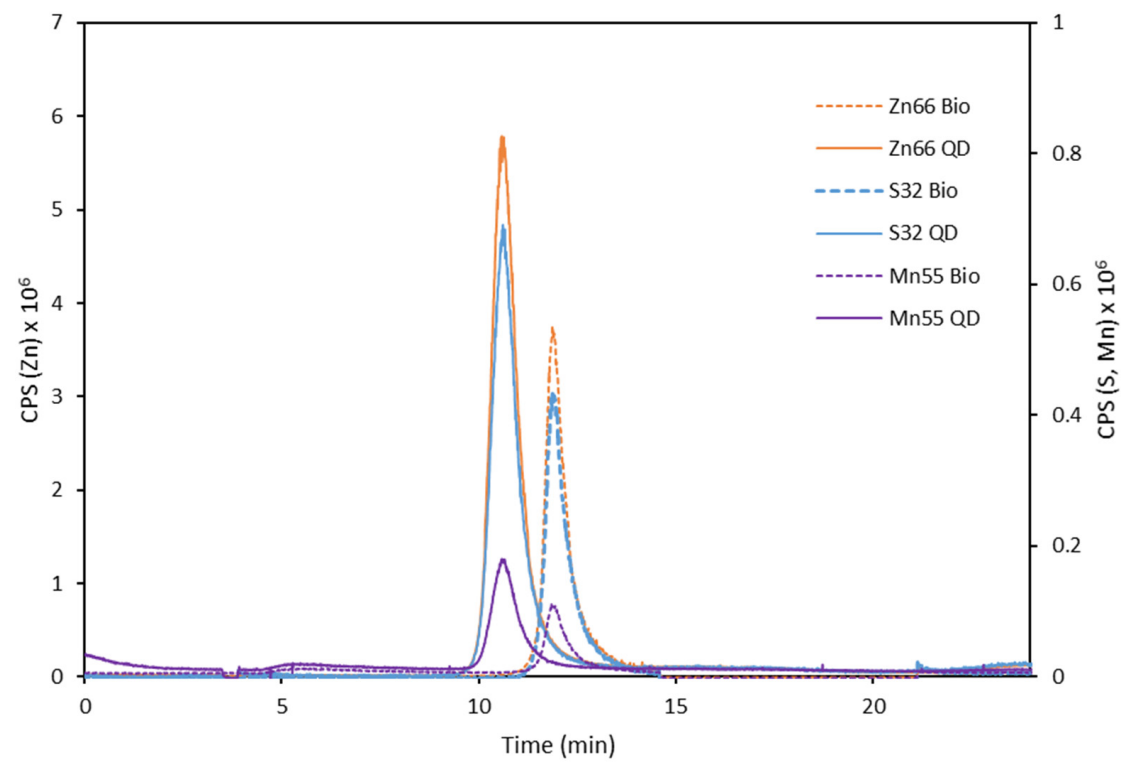

Figure 3. Elemental profiles obtained by asymmetric flow field-flow fractionation coupled on-line to an ICP-MS/MS (AF4-ICP-MS/MS). Zn66 Bio and Zn66 QD corresponds to the fractograms obtained, measuring the isotope 66 of Zn. S32 Bio and S32 QD corresponds to the fractograms obtained for the QD-Antibody bioconjugate and the QD respectively, measuring the isotope 32 of S. Mn55 Bio and Mn55 QD corresponds to the fractograms obtained for the QD-Antibody bioconjugate and the QD respectively, measuring the isotope 55 of Mn. Orange, ${ }^{64} \mathrm{Zn}^{+} \rightarrow{ }^{64} \mathrm{Zn}^{+}$; purple, ${ }^{55} \mathrm{Mn}^{+} \rightarrow{ }^{55} \mathrm{Mn}^{+}$; blue, ${ }^{32} \mathrm{~S}^{+} \rightarrow{ }^{48} \mathrm{SO}^{+}$. 
Elemental characterization of the QDs-bioconjugates by AF4-ICP-MS/MS was carried out to assess the succeed on the bioconjugation process. Measurement of the elemental area ratios in the fractographic peaks showed that while the $\mathrm{Zn} / \mathrm{Mn}$ ratio stays unaltered both for the free QDs and for the Ab-QDs ( $33 \pm 1$ and $32 \pm 1$, respectively), the Zn/S ratio changes from $8.3 \pm 0.2$ for the QDs to $7.2 \pm 0.4$ for the Ab-QD due to the contribution of sulfur present in the antibodies. This difference in $S$ element brings to light the actual conjugation of the QDs to the antibodies. Additionally, DLS measurements of the Ab-QD product were performed showing a higher hydrodynamic radius than that of the free colloidal QDs (See Supplementary Figure S2), which is in agreement with the results based on the elemental area ratios measurement.

\subsection{Evaluation of an Indirect Immunoassay Format for AFM1 Quantification}

Two different immunoassay schemes were compared for the development of the competitive phosphorescent immunosensor for sensitive AFM1 quantification in bovine milk. One of the formats was based on an indirect immunoassay, in which a derivative of the antigen AFM1-BSA was labelled with the d-QDs. In this approach, the wells of the microtiter plates were coated with anti-AFM1 antibodies following the procedure described in Section 2.3.3. The competitive immunoassay was based on an incubation of such microtiter plates with aqueous standards of AFM1 at different concentration levels (or milk samples containing AFM1) and QD-labelled AFM1-BSA antigen.

Figure 4 shows the analytical signal (phosphorescence spectra) recorded during the analysis of a blank and two samples containing different concentrations of AFM1 ( $0.05 \mathrm{ng} \mathrm{mL}^{-1}$ and $50 \mathrm{ng} \mathrm{mL}^{-1}$, respectively) under the optimized conditions of the assay (e.g., BSA-AFM1, antibody and BSA blocking solutions concentrations, analysis times, washing and blocking procedures, etc.).

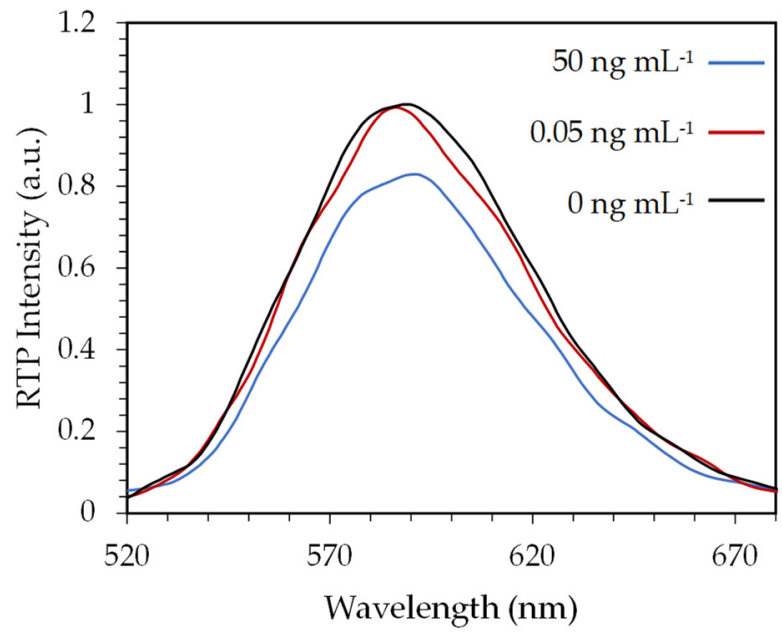

Figure 4. Phosphorescence emission signals registered after analysis of samples containing different AFM1 concentrations employing the indirect competitive immunoassay.

As expected, the RTP intensity measured decreases with increasing AFM1 concentration in the sample. This behavior results from the competition between the free AFM1 present in the sample and the QDs-labelled AFM1 added in the assay by the limited number of recognition sites of the antibodies coating the wells of the microtiter plate. Thus, an increase in the concentration of AFM1 in the sample results in a smaller amount of QDs-labelled AFM1 that will be retained by the immobilized antibodies and, therefore, the RTP signal detected will be lower.

Unfortunately, after optimization of the AFM1-BSA-QDs bioconjugation protocol and the immunoassay experimental parameters, the sensitivity achieved was not good enough to quantify AFM1 in milk at the concentration levels required. As it can be seen in Figure 4, analytical signals recorded from the analysis of a blank solution (in the absence of AFM1) 
and from the analysis of a sample containing an AFM1 concentration of $0.05 \mathrm{ng} \mathrm{mL} \mathrm{mL}^{-1}$ (the maximum AFM1 concentration allowed in milk) are statistically undistinguishable. When AFM1 concentration in the sample was increased up to $50 \mathrm{ng} \mathrm{mL}{ }^{-1}$, the analytical signal was clearly different from that of a blank.

Such poor analytical performance observed in this indirect assay could be most probably derived from significant steric impediments in the formation of the immunocomplex. Such steric effects are expected to occur due to the large size of the QD-BSA-AFM1 conjugate as compared to the free AFM1 of the standards (i.e., the smaller size of the free aflatoxin molecule could facilitate its binding to the supported antibody preferably from the engineered QD-labelled analyte).

\subsection{Evaluation of a Direct Immunoassay Format for AFM1 Quantification}

Alternatively, a direct immunoassay scheme, based on the conjugation of colloidal water-soluble Mn-doped ZnS QDs to an anti-IgG antibody was investigated (see Figure 1B). Therefore, a bioconjugation of the antibody with the luminescent QDs, as labels, was needed.

After the optimization of the bioconjugation and the experimental conditions, the performance characteristics of such an RTP immunoassay were investigated. Figure 5A collects the spectra obtained for different AFM1 concentrations, ranged from 0.005 to $50 \mathrm{ng} \mathrm{mL}{ }^{-1}$. All the standard solutions were prepared in PBS $10 \mathrm{mM} \mathrm{pH} \mathrm{7.4.}$

It was observed that the RTP intensity measured decreases when increasing AFM1 concentration in the sample. An increase in the AFM1 levels present in the sample or standards will complex an increased number of free anti-AFM1 antibodies during the pre-incubation stage. In this way, the number of free AFM1 antibodies available to interact with the AFM1 coating the well of the microtiter plate will be lower. As there is a lower number of anti-AFM1 antibodies retained in the well of the microtiter plate, the number of QD-labeled anti-IgG antibodies that will interact, and therefore that will be retained at the end of the assay, will also be lower, resulting in a decrease in the RTP signal.

Figure 5B shows the linear response between RTP peak intensity (measured at $590 \mathrm{~nm}$ ) and the logarithm value of AFM1 concentration, obtained from the calibration curve with the direct phosphorescent immunoassay developed. Obtained regression equation is shown in Equation (1).

$$
\mathrm{I}=-0.3541 \cdot \ln \mathrm{C}+7.9693
$$

where I is the peak intensity of RTP at $590 \mathrm{~nm}, \mathrm{C}$ is the concentration of AFM1.

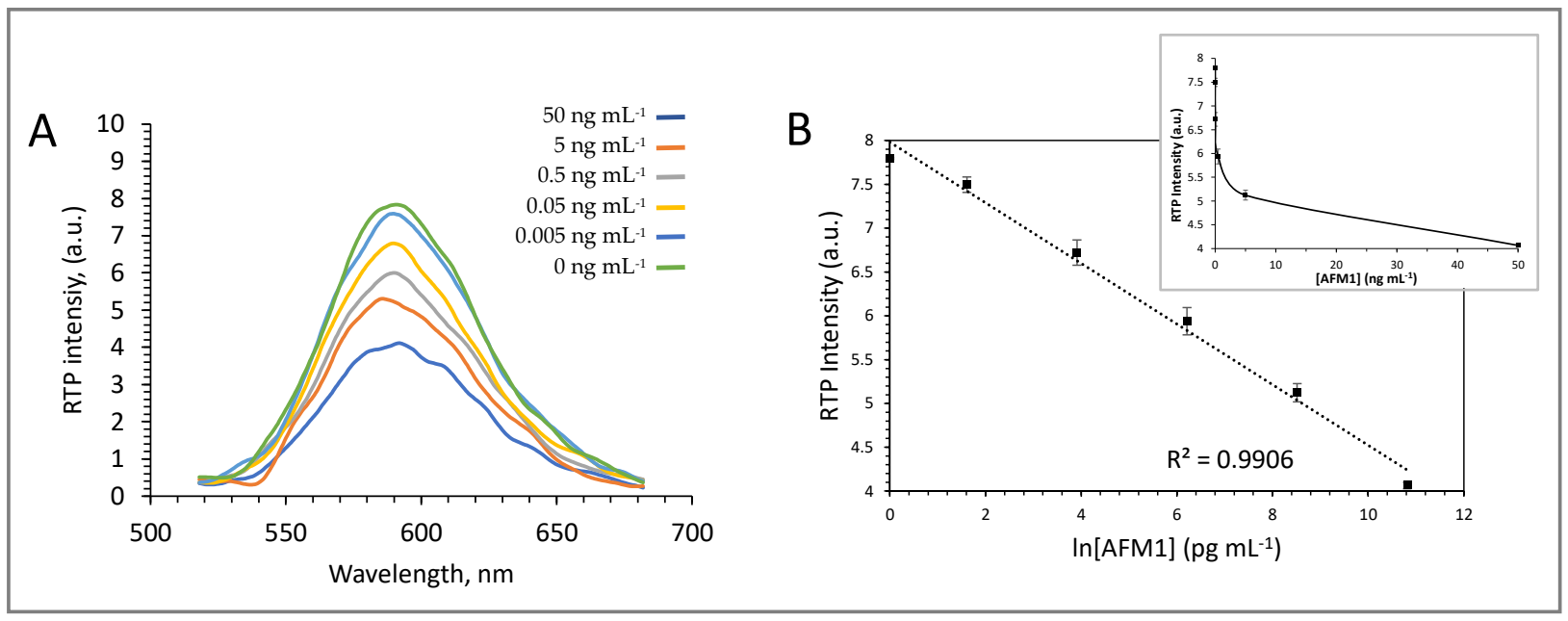

Figure 5. (A) RTP spectra obtained for the AFM1 concentrations assayed; (B) Immunoassay response curve linearized by applying $\ln$ to the $\mathrm{x}$-axis at different AFM1 concentrations. Inset: non-linearized immunoassay response curve. 
The characterization of the method in the basis of sensibility, precision and linear range has been carried out and obtained values are collected in Table 1 . The limit of detection (LOD) signal corresponds to the analytical signal of the blank $\left(\mathrm{S}_{\mathrm{b}}\right)$ minus three times the standard deviation from the blank signal $\left(\sigma_{\mathrm{b}}\right)$ and has reached a value of $0.002 \mathrm{ng} \mathrm{mL}{ }^{-1}$. The minimum concentration of AFM1 detected in this proposed assay has been $0.004 \mathrm{ng} \mathrm{mL}^{-1}$ AFM1, and this experimental value has been established as limit of quantification (LOQ). These values are 23 and 12 times respectively, lower than the Maximum Residue Limit $\left(\mathrm{MLR}=0.05 \mathrm{ng} \mathrm{mL}{ }^{-1}\right)$ set by the EU for AFM1 in raw milk, heat-treated milk and milk for the manufacture of milk-based products (Official Journal of the European Union, 2010). The linear range was assayed from LOQ to $50 \mathrm{ng} \mathrm{mL}^{-1}$.

Table 1. Analytical parameters of the QD-based immunoassay for AFM1 quantification.

\begin{tabular}{cc}
\hline Parameter & \\
\hline Limit of detection (LOD) $\left(\mathrm{ng} \mathrm{mL}^{-1}\right)$ & 0.002 \\
Limit of quantification (LOQ) $\left(\mathrm{ng} \mathrm{mL}^{-1}\right)$ & 0.004 \\
Coefficient of variation, $\mathrm{CV}(\%) n=5$ & 6 \\
Linear range $\left(\mathrm{ng} \mathrm{mL} \mathrm{mL}^{-1}\right)$ & LOQ-50 \\
\hline $\mathrm{CV}=$ standard deviation $/$ mean $(n=5)$ \\
\hline
\end{tabular}

Finally, the performance of the phosphorescent QD-based immunoassay was validated for analysis of cow milk enriched with AFM1. Two different skimmed milk samples were spiked with AFM1 at concentrations well below $\left(0.005 \mathrm{ng} \mathrm{mL}^{-1}\right)$ and the EU limits levels (MLR of FDA, $0.5 \mathrm{ng} \mathrm{mL}^{-1}$ ). As only pre-treatment, milk samples were diluted 10 times in $10 \mathrm{mM}$ PBS

The experimentally determined AFM1 concentrations are given in Table 2 and were very close to the theoretical values in the samples assayed: the mean recovery for AFM1 ranged from 81 to $90 \%$ with the standard error in the range of 3-14\% toward the spiked levels. As expected, RTP signal decreases when AFM1 content in the milk increased. Moreover, for low RTP signals an increase of uncertainty of the final measure is observed. Similarly, when the AFM1 content is lower (e.g., $0.005 \mathrm{ng} \mathrm{mL}^{-1}$ ) the RTP signal measured is higher, and the error in the measurement becomes minimum.

Table 2. Recoveries for AFM1 spiked milk samples $(n=3)$.

\begin{tabular}{|c|c|c|c|c|}
\hline \multirow{2}{*}{ Sample } & \multicolumn{2}{|c|}{ AFM1 Concentration $\left(\mathrm{pg} \mathrm{mL}^{-1}\right)$} & \multirow{2}{*}{ Recovery (\%) } & \multirow{2}{*}{ RSD (\%) } \\
\hline & Added & Experimental & & \\
\hline Skimmed milk & 5 & 4.08 & 81 & 3 \\
\hline Skimmed milk & 500 & 453 & 90 & 14 \\
\hline
\end{tabular}

\section{Conclusions}

A competitive immunosensor for sensitive quantification of food toxins which makes use of the advantages of the phosphorescence detection provided by Mn-doped QDs used as antibodies tags is here proposed.

The comparison of the analytical parameters derived from the doses-response curves obtained for the two immunoassays configurations assayed (direct and indirect formats) revealed a better detection limit for the format based on the conjugation of the waterstabilized phosphorescent QDs to a secondary anti-IgG antibody. Such direct competitive immunoassay was successfully developed and exhibited good analytical features for the sensitive quantification of AFM1 in milk, being a highly valuable alternative to conventional techniques for the quantification of aflatoxin M1 in milk.

The proposed immunoassay is an environmentally friendly, robust, quick and low-cost methodology. The calibration statistics and validation of this procedure with skimmed milk samples have demonstrated its compliance with the EU legislation limits for the maximum allowed AFM1 concentration in milk products. Moreover, the simplicity of the sample 
pre-treatment of the raw milk allows the proposed procedure to be carried out on-site by using portable luminescence instruments for collecting the final RTP signal.

In brief, we have demonstrated the feasibility of the use of Mn:ZnS QDs as highly valuable phosphorescent labels in the development of a quantitative immunoassay for sensitive AFM1 detection in milk samples (a demand of high interest in food quality control). In addition, it is important to point out that it was not necessary to perform any complex sample pre-treatment and an aqueous sample dilution is only required for the success of the quantification.

It is envisaged that the competitive phosphorescent immunoassays format developed here have wide potential applicability in different areas such as bioscience, food analysis, clinical settings, etc., just by selecting an adequate selective receptor specific of the desired analyte, making it possible to expand the applicability to other relevant problems. Although in the present study AFM1 was used as a target analyte, the approach here developed (based on the labelling of a secondary antibody with the phosphorescent QDs) can be directly translated for detection or quantification of any other environmental, clinical or food relevant small molecules by just selecting an appropriate capture primary antibody.

Supplementary Materials: The following are available online at https: / www.mdpi.com/article/ 10.3390/ chemosensors10020041/s1, Figure S1: Dynamic Light Scattering spectrum of DHLA MN:ZnS QDs $(n=3)$, Figure S2: Dynamic Light scattering spectrum of DHLA Mn:ZnS QDs (red line) and QD-Ab conjugate (green line), Figure S3: RTP Spectra corresponding to different bioconjugates obtained at different QD: Ab ratios. Table S1: Asymmetric Flow Field Flow Fractionation (AF4) operation conditions.

Author Contributions: Conceptualization, J.M.C.-F., L.J.R. and A.S.; methodology, S.F., A.S.-V. and C.M.; validation, S.F., M.M.-M. and A.S.; formal analysis, J.M.C.-F., L.J.R. and A.S.; investigation, S.F., A.S.-V., C.M.; resources, A.S., L.J.R. and J.M.C.-F.; data curation, S.F., M.M.-M. and A.S.; writing-original draft preparation, S.F.; writing—review and editing, M.M.-M., J.M.C.-F. and A.S.; supervision, A.S., L.J.R. and J.M.C.-F.; funding acquisition, A.S. and J.M.C.-F. All authors have read and agreed to the published version of the manuscript.

Funding: This research was funded by Spanish Ministry of Science and Innovation (PID2020117282RB-I00 and PID2019-109698GB-I00), Principado de Asturias Regional Government (projects IDI/2021/000081 and IDI/2021/000102) co-financed by the European Union through the ERDF (European Regional Development Fund), FPI fellowship BES-2017-081314 supported by MCIN/AEI/10.13039/ 501100011033 and ESF "El FSE invierte en tu futuro".

Conflicts of Interest: The authors declare no conflict of interest.

\section{References}

1. European Commission. Regulation (EU) No. 165/2010. Off. J. EU 2010, L50, 8-12.

2. Min, L.; Fink-Gremmels, J.; Li, D.; Tong, X.; Tang, J.; Nan, X.; Yu, Z.; Chen, W.; Wang, G. An overview of aflatoxin B1 biotransformation and aflatoxin M1 secretion in lactating dairy cows. Anim. Nutr. 2021, 7, 42-48. [CrossRef] [PubMed]

3. Saha Turna, N.; Wu, F. Aflatoxin M1 in milk: A global occurrence, intake, \& exposure assessment. Trends Food Sci. Technol. 2021, 110, 183-192. [CrossRef]

4. Yao, H.; Hruska, Z.; Diana Di Mavungu, J. Developments in detection and determination of aflatoxins. World Mycotoxin J. 2015, 8, 181-191. [CrossRef]

5. Salari, N.; Kazeminia, M.; Vaisi-Raygani, A.; Jalali, R.; Mohammadi, M. Aflatoxin M1 in Milk Worldwide from 1988 to 2020 : A Systematic Review and Meta-Analysis. J. Food Qual. 2020, 2020, 8862738. [CrossRef]

6. FDA US. Action Levels for Poisonous or Deleterious Substances in Human Food and Animal Feed; Food and Drug Administration: Silver Spring, MD, USA, 2000.

7. Wood, J.E.; Gill, B.D.; Indyk, H.E.; Rhemrev, R.; Pazdanska, M.; Wood, J. Determination of Aflatoxin M1 in Liquid Milk, Cheese and Selected Milk Proteins by Automated Online Immunoaffinity Cleanup with Liquid Chromatography-Fluorescence Detection. J. AOAC Int. 2021, 104, 719-724. [CrossRef]

8. Hashemi, M.; Taherimaslak, Z. Determination of aflatoxin M1 in liquid milk using high performance liquid chromatography with fluorescence detection and magnetic solid phase extraction. RSC Adv. 2014, 4, 33497-33506. [CrossRef] 
9. Andrade, P.D.; da Silva, J.L.G.; Caldas, E.D. Simultaneous analysis of aflatoxins B1, B2, G1, G2, M1 and ochratoxin A in breast milk by high-performance liquid chromatography/fluorescence after liquid-liquid extraction with low temperature purification (LLE-LTP). J. Chromatogr. A 2013, 1304, 61-68. [CrossRef]

10. González-Jartín, J.M.; Rodríguez-Cañás, I.; Alfonso, A.; Sainz, M.J.; Vieytes, M.R.; Gomes, A.; Ramos, I.; Botana, L.M. Multianalyte method for the determination of regulated, emerging and modified mycotoxins in milk: QuEChERS extraction followed by UHPLC-MS/MS analysis. Food Chem. 2021, 356, 129647. [CrossRef]

11. Bognanno, M.; La Fauci, L.; Ritieni, A.; Tafuri, A.; De Lorenzo, A.; Micari, P.; Di Renzo, L.; Ciappellano, S.; Sarullo, V.; Galvano, F. Survey of the occurrence of Aflatoxin M1 in ovine milk by HPLC and its confirmation by MS. Mol. Nutr. Food Res. 2006, 50, 300-305. [CrossRef]

12. Zhou, J.; Xu, J.J.; Cong, J.M.; Cai, Z.X.; Zhang, J.S.; Wang, J.L.; Ren, Y.P. Optimization for quick, easy, cheap, effective, rugged and safe extraction of mycotoxins and veterinary drugs by response surface methodology for application to egg and milk. J. Chromatogr. A 2018, 1532, 20-29. [CrossRef] [PubMed]

13. Beloglazova, N.V.; Shmelin, P.S.; Goryacheva, I.Y.; De Saeger, S. Liposomes loaded with quantum dots for ultrasensitive on-site determination of aflatoxin M1 in milk products Rapid Detection in Food and Feed. Anal. Bioanal. Chem. 2013, 405, 7795-7802. [CrossRef] [PubMed]

14. Gan, N.; Zhou, J.; Xiong, P.; Hu, F.; Cao, Y.; Li, T.; Jiang, Q. An ultrasensitive electrochemiluminescent immunoassay for aflatoxin M1 in milk, based on extraction by magnetic graphene and detection by antibody-labeled CdTe quantum dots-carbon nanotubes nanocomposite. Toxins 2013, 5, 865-883. [CrossRef] [PubMed]

15. Zhou, Y.; Xiong, S.; Zhang, K.K.; Feng, L.; Chen, X.; Wu, Y.; Huang, X.; Xiong, Y. Quantum bead-based fluorescence-linked immunosorbent assay for ultrasensitive detection of aflatoxin M 1 in pasteurized milk, yogurt, and milk powder. J. Dairy Sci. 2019, 102, 3985-3993. [CrossRef]

16. Díaz-González, M.; de la Escosura-Muñiz, A.; Fernandez-Argüelles, M.T.; García Alonso, F.J.; Costa-Fernandez, J.M. Quantum Dot Bioconjugates for Diagnostic Applications. Top. Curr. Chem. 2020, 378, 133-176. [CrossRef]

17. Hildebrandt, N.; Spillmann, C.M.; Russ Algar, W.; Pons, T.; Stewart, M.H.; Oh, E.; Susumu, K.; Díaz, S.A.; Delehanty, J.B.; Medintz, I.L. Energy transfer with semiconductor quantum dot bioconjugates: A versatile platform for biosensing, energy harvesting, and other developing applications. Chem. Rev. 2017, 117, 536-711. [CrossRef]

18. Sotelo-Gonzalez, E.; Roces, L.; Garcia-Granda, S.; Fernandez-Arguelles, M.T.; Costa-Fernandez, J.M.; Sanz-Medel, A. Influence of $\mathrm{Mn}^{2+}$ concentration on $\mathrm{Mn}^{2+}$-doped $\mathrm{ZnS}$ quantum dot synthesis: Evaluation of the structural and photoluminescent properties. Nanoscale 2013, 5, 9156-9161. [CrossRef]

19. Wu, P.; Yan, X.P. Doped quantum dots for chemo/biosensing and bioimaging. Chem. Soc. Rev. 2013, 42, 5489-5521. [CrossRef]

20. Garcia-Cortes, M.; Sotelo González, E.; Fernández-Argüelles, M.T.; Encinar, J.R.; Costa-Fernández, J.M.; Sanz-Medel, A. Capping of Mn-Doped ZnS Quantum Dots with DHLA for Their Stabilization in Aqueous Media: Determination of the Nanoparticle Number Concentration and Surface Ligand Density. Langmuir 2017, 33, 6333-6341. [CrossRef]

21. Kolmykov, O.; Coulon, J.; Lalevée, J.; Alem, H.; Medjahdi, G.; Schneider, R. Aqueous synthesis of highly luminescent glutathionecapped $\mathrm{Mn}^{2+}$-doped $\mathrm{ZnS}$ quantum dots. Mater. Sci. Eng. C 2014, 44, 17-23. [CrossRef]

22. Wang, Y.; Liang, X.; Ma, X.; Hu, Y.; Hu, X.; Li, X.; Fan, J. Simple and greener synthesis of highly photoluminescence Mn ${ }^{2+}$-doped ZnS quantum dots and its surface passivation mechanism. Appl. Surf. Sci. 2014, 316, 54-61. [CrossRef]

23. García-Cortés, M.; Fernández-Argüelles, M.T.; Costa-Fernández, J.M.; Sanz-Medel, A. Sensitive prostate specific antigen quantification using dihydrolipoic acid surface-functionalized phosphorescent quantum dots. Anal. Chim. Acta 2017, 987, 118-126. [CrossRef]

24. Zhang, Z.; Miao, Y.; Zhang, Q.; Yan, G. Facile and sensitive detection of protamine by enhanced room-temperature phosphorescence of Mn-doped ZnS quantum dots. Anal. Biochem. 2015, 478, 90-95. [CrossRef]

25. Bian, W.; Wang, F.; Wei, Y.; Wang, L.; Liu, Q.; Dong, W.; Shuang, S.; Choi, M.M.F. Doped zinc sulfide quantum dots based phosphorescence turn-off/on probe for detecting histidine in biological fluid. Anal. Chim. Acta 2015, 856, 82-89. [CrossRef]

26. Ban, R.; Zhu, J.J.; Zhang, J. Manganese-doped ZnS quantum dots as a phosphorescent probe for use in the bi-enzymatic determination of organophosphorus pesticides. Microchim. Acta 2014, 181, 1591-1599. [CrossRef]

27. Tan, L.; Kang, C.; Xu, S.; Tang, Y. Selective room temperature phosphorescence sensing of target protein using Mn-doped ZnS QDs-embedded molecularly imprinted polymer. Biosens. Bioelectron. 2013, 48, 216-223. [CrossRef] 